\title{
Morphological and Magnetic Properties of Superparamagnetic Carbon-Coated Fe Nanoparticles Produced by Arc Discharge
}

\author{
N. Aguiló-Aguayo*, M. J. Inestrosa-Izurieta, J. García-Céspedes, and E. Bertran \\ FEMAN Group, IN2UB, Dep. Física Aplicada i Òptica, Universitat de Barcelona, \\ Martí i Franquès, 1, E08028 Barcelona, Spain
}

\begin{abstract}
Spherical carbon coated iron particles of nanometric diameter in the 5-10 nm range have been produced by arc discharge at near-atmospheric pressure conditions (using 5-8.104 $\mathrm{Pa}$ of $\mathrm{He}$ ). The particles exhibit a crystalline dense iron core with an average diameter $7.4 \pm 2.0 \mathrm{~nm}$ surrounded by a sealed carbon shell, shown by transmission electron microscopy (TEM), selected-area diffraction (SAED), energy-dispersive X-ray analysis (STEM-EDX) and electron energy loss spectroscopy (EELS). The SAED, EDX and EELS results indicate a lack of traces of core oxidized phases showing an efficient protection role of the carbon shell. The magnetic properties of the nanoparticles have been investigated in the $5-300 \mathrm{~K}$ temperature range using a superconducting quantum interference device (SQUID). The results reveal a superparamagnetic behaviour with an average monodomain diameter of $7.6 \mathrm{~nm}$ of the nanoparticles. The zero field cooled and field cooled (ZFC-FC) magnetization curves show a blocking temperature $\left(T_{\mathrm{B}}\right)$ at room temperature very suitable for biomedical applications (drug delivery, magnetic resonance imaging-MRI-, hyperthermia).
\end{abstract}

Keywords: Superparamagnetism, Behaviour, Nanoparticles, Monodomain, Arc Discharge.

\section{INTRODUCTION}

In the recent years, superparamagnetic particles have had an important role due to their unique magnetic properties in the modern medical field for several applications, such as drug delivery, magnetic resonance imaging-MRI-and hyperthermia among other cancer curing. ${ }^{1}$ The superparamagnetic behaviour is revealed under a critical size for a monodomain nanoparticle, which depends on the saturation magnetization and the magneto-crystalline anisotropy of the material. In addition, nanoparticles with spherical shape are more suitable for applications in the human body. ${ }^{2}$ In case of carbon coated iron nanoparticles, the carbon shell protects nanoparticles from oxidation which could help to maintain higher magnetic responses and making the system biocompatible with the human body.

In this article, the structure and morphologic characteristics about shape and size distribution of carbon-coated iron-nanoparticles $(\mathrm{Fe} @ \mathrm{C})$ have been studied using transmission electron microscopy (TEM) and selected area electron diffraction (SAED). Besides, the absence of oxygen in the nanoparticles has been determined by energy-dispersive

*Author to whom correspondence should be addressed.
X-ray analysis (STEM-EDX) and electron energy loss spectroscopy (EELS). Furthermore, in order to establish relationships between magnetic behaviour and morphological and structure characteristics, the superparamagnetic characteristics of $\mathrm{Fe} @ \mathrm{C}$ nanoparticles have been investigated.

\section{EXPERIMENTAL DETAILS}

The arc discharge apparatus used in this experiment is based on a vertical carbon rod $(50 \mathrm{~mm}$ in length and $12 \mathrm{~mm}$ in diameter) as the cathode (99.9\% purity). Multiple foils of iron (purity 99.99\%) as raw material were tightly fixed in a hole of the graphite column which is grounded and used as the anode. A modification of the arc discharge reactor allows a particle manipulation procedure at pristine conditions. The nanoparticles can be directly collected and dispersed in deionised water without exposition to the atmosphere. Pure He was used as the source of plasma. The He pressure of the reaction chamber was set in the range of 5-8 $10^{4} \mathrm{~Pa}$, near-atmospheric pressure conditions. The current applied on the electrode was $40 \mathrm{~A}$, the voltage was about $125 \mathrm{~V}$ and the discharge process was maintained for 5-10 minutes. 
A Philips CM30 operating at $300 \mathrm{kV}$ was used for TEM and SAED structural characterization. STEM-EDX and EELS analysis were obtained by a TEM operating at $200 \mathrm{kV}$, which is equipped with a JEOL JEM $2010 \mathrm{~F}$ field emission gun. A superconducting-quantuminterference device (SQUID) magnetometer was used to study magnetic behaviour of carbon coated iron nanoparticles in the temperature range $5-300 \mathrm{~K}$ and using fields up to $55 \mathrm{kOe}$.

\section{RESULTS AND DISCUSSION}

The TEM images of carbon coated iron nanoparticles $(\mathrm{Fe} @ \mathrm{C})$ indicate that the presence of nanoparticles with a spherical shape and carbon encapsulation of $3-5 \mathrm{~nm}$ is confirmed (Fig. 1). The SAED pattern referring to Fe@C nanoparticles (inset Fig. 1) demonstrates their crystalline structure. The electron diffraction pattern is identified as $\alpha$-Fe in the [11-1] zone axis, which shows that the core nanophases are in the form of $\alpha$-Fe (bcc).

The size distribution of the iron core of nanoparticles is determined from multiple TEM images (248 total population). The diameters are sorting into a histogram and data histogram are satisfactorily distributed following a function $f(D)$ which is described by a logarithmic-lineal distribution $^{3}$

$$
f(D)=\frac{1}{\sqrt{2 \pi} \sigma D} \exp \left\{-\frac{\ln ^{2}\left(D / D_{0}\right)}{2 \sigma^{2}}\right\}
$$

where $D_{0}$ is the most probable particle diameter and $\sigma$ is the standard deviation of $\ln (D)$. The average iron core diameter is $7.4 \mathrm{~nm}$ with a standard deviation of $2.0 \mathrm{~nm}$ (Fig. 2) in agreement with the following magnetic studies.

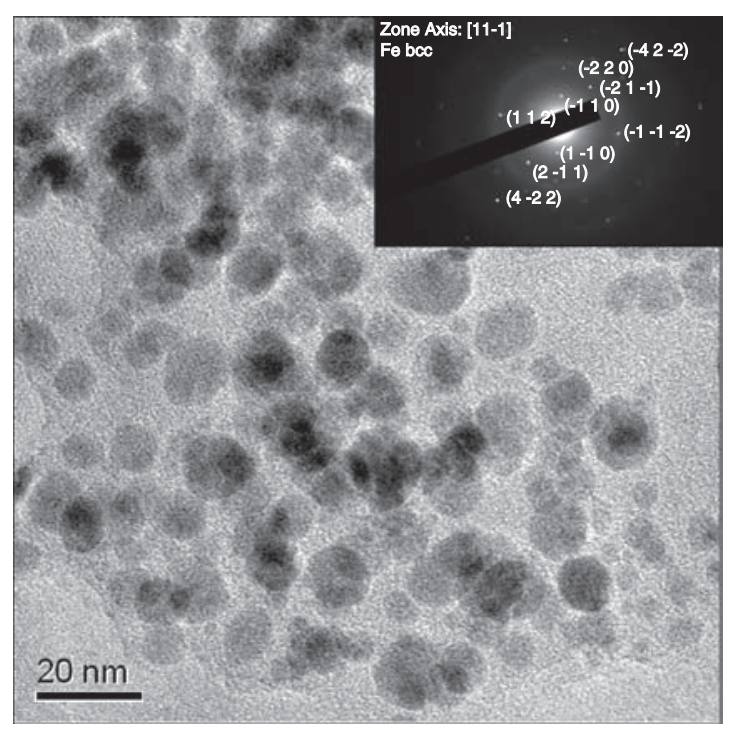

Fig. 1. TEM image of spherical iron nanoparticles encapsulated with a carbon shell of 3-5 nm thick. The SAED pattern in the inset of the figure reveals the crystalline structure of nanoparticles. The diffraction pattern corresponds to $\alpha$-Fe in the [11-1] zone axis.
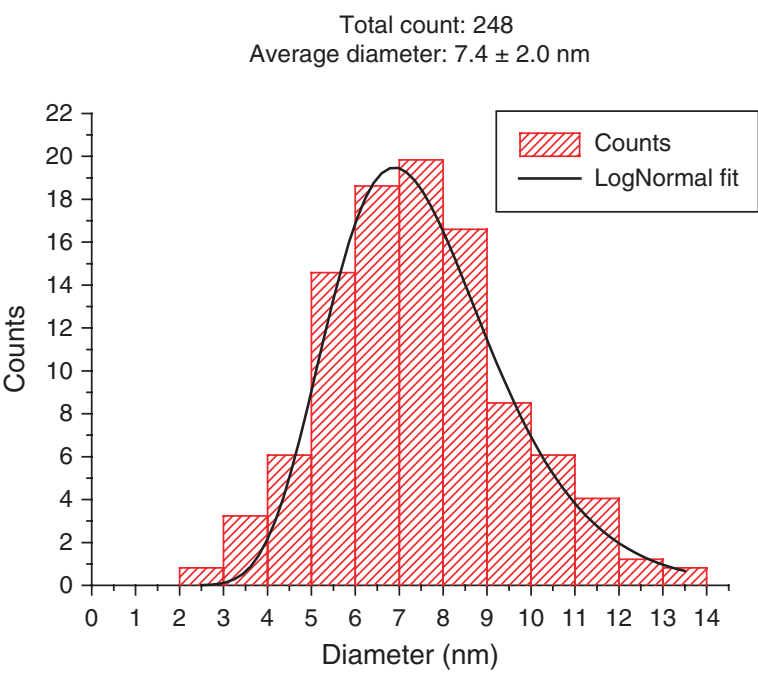

Fig. 2. Particle size distribution histogram of carbon coated iron nanoparticles. The carbon shell was not considered. The size of the iron core of the nanoparticles is following a logarithmic-linear distribution with an average iron core diameter of $7.4 \mathrm{~nm}$ in accordance with SQUID measurements.

In order to obtain more information about the elements present into nanoparticles, the electron probe was scanned along a region of the specimen and the intensity of the transmission electron signal was measured using detectors (STEM-EDX energy-dispersive X-ray analysis). The spectrum profile obtained from STEM-EDX analysis informs us about the presence of iron and carbon in the nanoparticles among other elements $(\mathrm{Si}, \mathrm{Cu}, \mathrm{Cl}, \mathrm{S})$ coming from the TEM grid, detectors or contamination (Fig. 3). Oxygen contamination is not observed in the spectrum profile which is in agreement with the no presence of iron oxides phases in the SAED pattern. This fact means iron core is completely sealed by carbon shell which protects nanoparticles from oxidation. In comparison with other techniques to produce core/shell nanoparticles, arc discharge technique can efficiently avoid oxidation of iron nanoparticles, which is a persistent problem to all forms of production of nanoscale iron particles. ${ }^{4}$

The composition map obtained by overlapping the $\mathrm{C}$ and Fe maps from electron energy loss spectroscopy (EELS) is shown in Figure 4. The green and red areas represent the

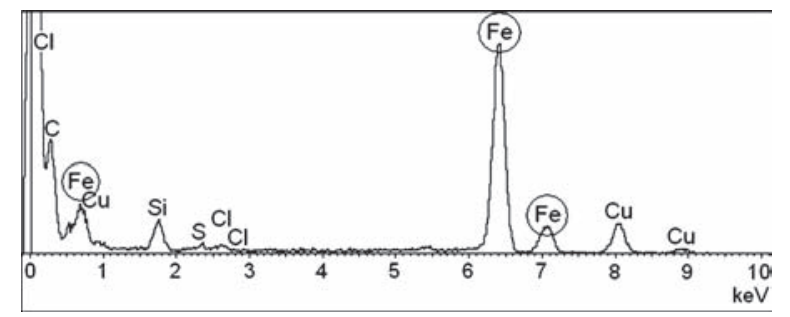

Fig. 3. STEM-EDX analysis of Fe@C nanoparticles. The absence of oxygen in the spectrum demonstrates the protection of the nanoparticles from oxidation due to the sealed carbon shell. 


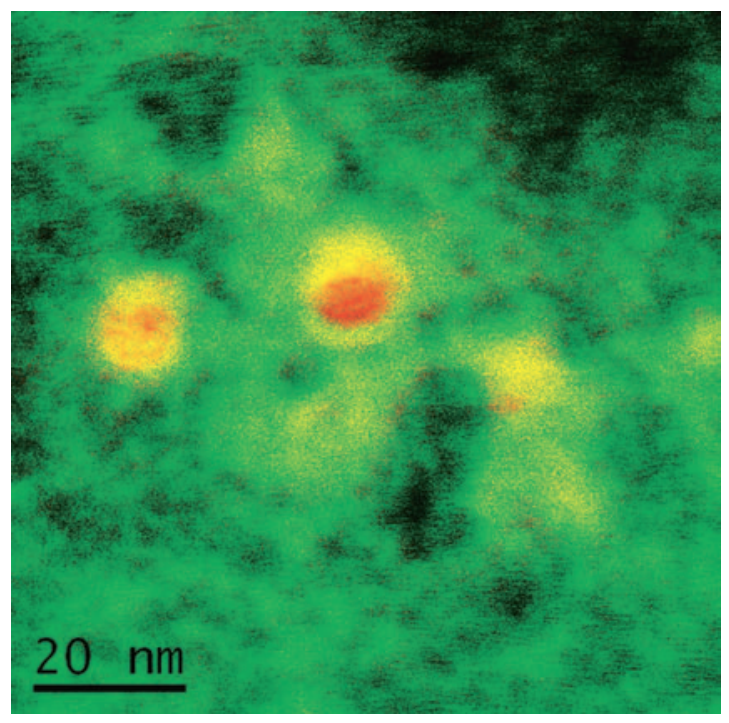

Fig. 4. Chemical map from Fe@C nanoparticles (red corresponds to the iron core distribution and green to the carbon distribution).

carbon and iron distributions, respectively. In accordance with SAED pattern and STEM-EDX analysis, there are no traces of oxygen in the elemental mapping.

Magnetic properties of $\mathrm{Fe} @ \mathrm{C}$ nanoparticles have been studied using (SQUID). The total mass of our sample was about $119 \mu \mathrm{g}$, this value was obtained oxidizing in air at $600{ }^{\circ} \mathrm{C}$ for $30 \mathrm{~min}$, converting all the carbon material into $\mathrm{CO}_{2}$. The remaining iron oxide was weighted and the iron content was extracted by considering all the material as $\mathrm{Fe}_{2} \mathrm{O}_{3}$. The corresponding saturation magnetization is $67 \mathrm{emu} / \mathrm{g}$ at $5 \mathrm{~K}$, which is lower than the saturation magnetization of $\alpha$-Fe bulk (212 emu/g) at $0 \mathrm{~K}$, however, is still higher than saturation magnetization of iron oxides $(10-50 \mathrm{emu} / \mathrm{g}){ }^{5}{ }^{5}$ The loss of magnetization as the particle size decrease depends largely on the crystalline magnetic anisotropy energy constant $K,{ }^{6}$ as well as, the coating

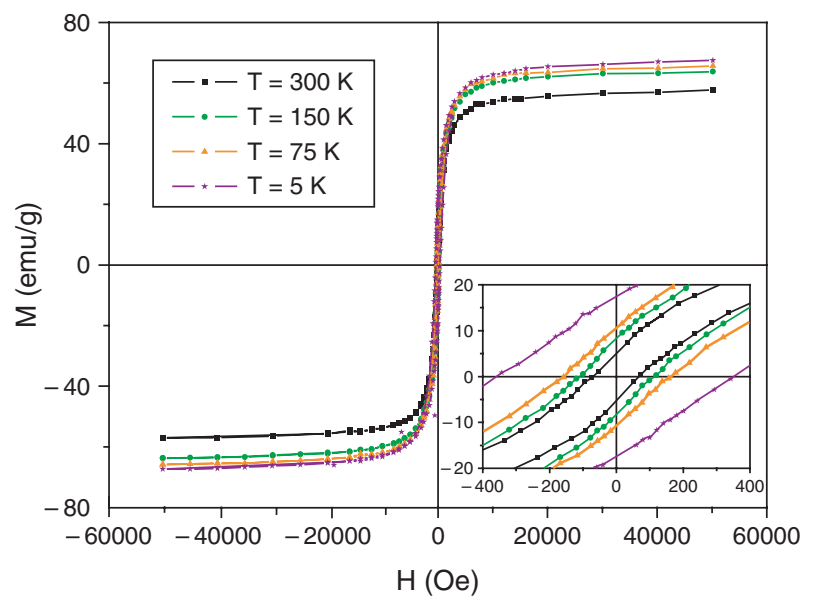

Fig. 5. Magnetization versus applied field at different temperatures $5 \mathrm{~K}$, $75 \mathrm{~K}, 150 \mathrm{~K}$, and $300 \mathrm{~K}$.

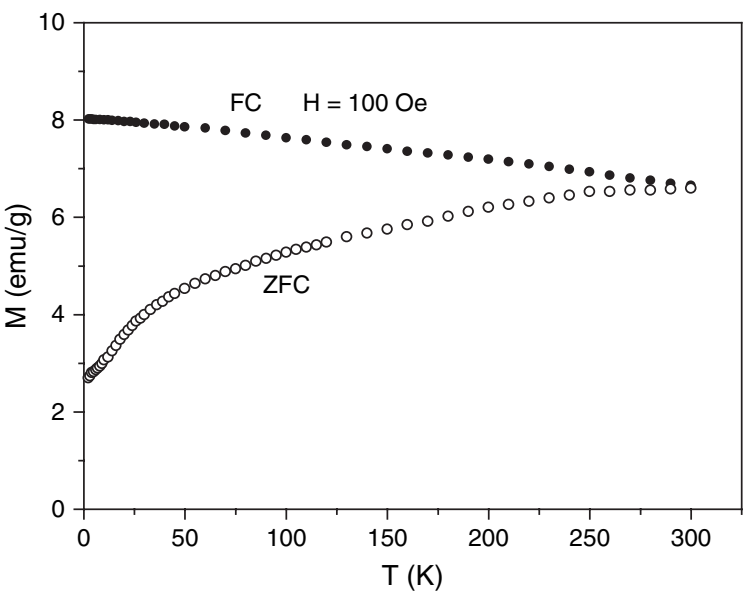

Fig. 6. Zero field cooled and field cooled magnetization curves (ZFC-FC) show the superparamagnetic behaviour of our nanoparticles above room temperature.

of particles with non-magnetic materials smay result in decrease in $M_{\mathrm{S}}$ values. $^{7}$

The magnetization $(M)$ versus the applied field $(H)$ at different temperatures $5,75,150$ and $300 \mathrm{~K}$ has been represented in Figure 5. The ratio of remnant to saturation magnetization at room temperature is $M_{\mathrm{r}} / M_{\mathrm{s}} \sim 0.08$, which clearly indicates that nanoparticles are in a superparamagnetic state. In addition, zero field cooled and field cooled (ZFC-FC) magnetization curves (Fig. 6) show a maximum at the blocking temperature $\left(T_{\mathrm{B}}\right)$, above this temperature which is around $300 \mathrm{~K}$ superparamagnetic behaviour is observed. ${ }^{8}$ Therefore, magnetization curve at room temperature can be fitted to a Langevin function ${ }^{9}$ (Fig. 7):

$$
\frac{M}{M_{\mathrm{S}}}=L\left(\frac{\mu H}{k_{\mathrm{B}} T}\right)=\operatorname{coth}\left(\frac{\mu H}{k_{\mathrm{B}} T}\right)-\frac{k_{\mathrm{B}} T}{\mu H}
$$

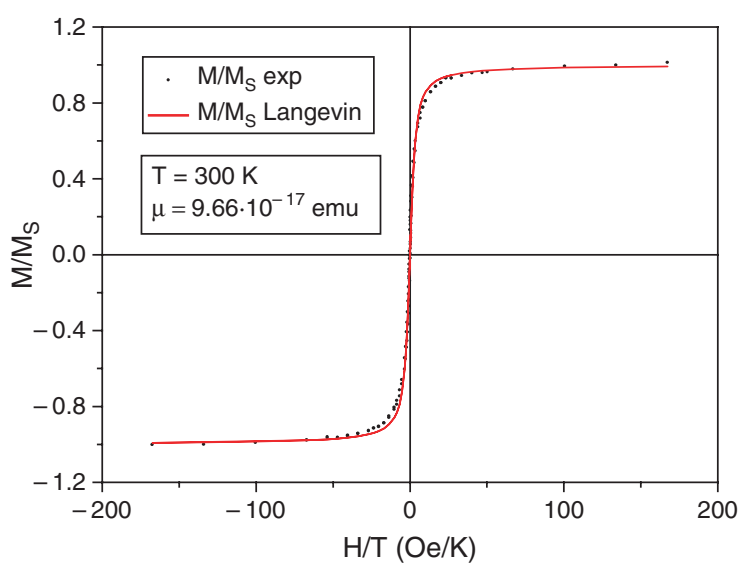

Fig. 7. Normalized magnetization versus $H / T$ at $300 \mathrm{~K}$ fitted to a Langevin function. The effective moment found was $\mu=9.66 \cdot 10^{-17} \mathrm{emu}$ corresponding to a domain size of spherical nanoparticles with a diameter about $7.6 \mathrm{~nm}$ in accordance to TEM observations. 
where $M_{\mathrm{S}}$ is the saturation magnetization, $\mu$ is the effective moment given by the product $M_{\mathrm{S}} \cdot\langle V\rangle$ and $\langle V\rangle$ is the average particle volume.

The magnetization curve fitting of Figure 7 to the Eq. (2) provided an effective moment value of $\mu \approx 9.66$. $10^{-17} \mathrm{emu}$, at a temperature of $300 \mathrm{~K}$. According to the SAED pattern, the nanoparticles core is mainly composed by $\alpha-\mathrm{Fe}$, which saturation magnetization at $0 \mathrm{~K}$ is $\sigma_{0}=$ $1740 \mathrm{emu} / \mathrm{cm}^{3}$. Using this value and the above determined effective moment, we have estimated a value $D=7.4 \mathrm{~nm}$ as the diameter, corresponding to the core of the spherical particles. This value has been confirmed by TEM measurements $(D=7.6 \pm 2.0 \mathrm{~nm})$. We suggest that the system is formed by monodomain nanoparticles about $\approx 7 \mathrm{~nm}$ with superparamagnetic behaviour.

\section{CONCLUSIONS}

Carbon coated iron nanoparticles (Fe@C) have been obtained by arc discharge technique. They consist of spherical nanoparticles, with an average iron core diameter of $7.6 \pm 2.0 \mathrm{~nm}$ (size distribution corresponds to a logarithmic-linear function) and a carbon shells thickness of 3-5 nm.

The crystalline structure of nanoparticles, provided by SAED, consists of iron cores of $\alpha$-Fe with non iron oxide phases present. Furthermore, STEM-EDX and EELS analysis of Fe@C nanoparticles confirmed the absence of oxygen, evidencing an efficient protection effect of the sealed carbon shell against the oxidation of the iron core.

The SQUID analysis of Fe@C nanoparticles has shown hysteretic and temperature dependent magnetic responses described by a superparamagnetic behaviour at temperatures above $300 \mathrm{~K}$. The diameter of the spherical particle domain obtained from the fitting magnetization curve at room temperature to the Langevin function is about $7 \mathrm{~nm}$ in accordance with TEM observations, which confirms the superparamagnetic character of the nanoparticles. The morphological and magnetical characteristics of the arc discharge Fe@C nanoparticles shown in this work are suitable for biomedical applications. These particles should be able to cross the blood brain barrier (BBB) and to be very suitable for drug delivery, magnetic resonance imaging and hyperthermia applications. Additional work will be done to demonstrate these points.

Acknowledgments: This study was supported by projects CSD2006-12 and DPI2006-03070 of MEDU of Spain and SGR2005-00666 of Generalitat de Catalunya. The authors thank D. N. Clos for the SQUID measurements and Serveis Científico-tècnics of the Universitat de Barcelona (SCT-UB) for measurements facilities.

\section{References and Notes}

1. D. Bahadur and G. Jyotsnendu, Sadhana, June/August (2003), Vol. 28, Parts 3 \& 4, pp. 639-656.

2. I. Nedkov, L. Slavov, T. Merodiiska, P. Lukanov, Ph. Tailhades, M. Gougeon, and R. E. Vandenberghe, J. Nanopart. Res. 10, 877 (2008).

3. X. Batlle and A. Labarta, J. Phys. D: Appl. Phys. 35, R16 (2002).

4. B. Ravel, E. E. Carpenter, and V. G. Harris, J. Appl. Phys. 91, 8195 (2002).

5. A. K. Gupta and M. Gupta, Biomaterials 26, 3995 (2005).

6. T. Sato, T. Iijima, M. Sekin, and N. Inagaki, J. Magn. Magn. Mater. 65, 252 (1987).

7. F. Tourinho, R. Franck, R. Massart, and R. Perzynski, Prog. Colloid. Polym. Sci. 79, 198 (1989).

8. D. L. Leslie-Pelecky and R. D. Rieke, Chem. Mater. 8, 1770 (1998).

9. M. E. McHenry, S. A. Majetich, J. O. Artman, M. DeGaef, and S. W. Staley, Phys. Rev. B 49, 11358 (1994).

Received: 10 December 2008. Accepted: 20 January 2009. 